\section{International Scientific Journal Theoretical \& Applied Science}

p-ISSN: 2308-4944 (print) e-ISSN: 2409-0085 (online)

Year: 2018 Issue: 09 Volume: 65

Published: $27.09 .2018 \quad$ http://T-Science.org

\section{Ilaha Nadır Orujova Reseacher, Azerbaijan Medical University Baku, Azerbaijan orucovailaha@rambler.ru}

Ajdar Sardar Asadov Ph.D., assistant of Diagnostic radiology and radiotherapy Department Azerbaijan Medical University Baku, Azerbaijan doktor.asadov@gmail.com

SECTION 20. Medicine.

Bahadur Alekber Baxshiev

M.D., professor of Diagnostic radiology and radiotherapy Department Azerbaijan Medical University, Baku, Azerbaijan

\title{
DIAGNOSTIC VALUE OF SOME PARAMETERS OF RADIOLOGY METHODS IN BREAST CANCER
}

\begin{abstract}
The aim of our work was to determine the diagnostic value of radiology methods used to detect breast cancer. In this study, we presented the results of a study of 76 women (18 - 79 years) who underwent a breast cancer examination in the Oncological Clinic of the Azerbaijan Medical University during the period from 2014 to 2017. All patients underwent ultrasound with a combination of doppler and mammography. Subsequently, out of 76 examined patients, 48 women had malignant tumors and 28 had benign neoplasms. Considering account the results of our studies, it can be inferred that when evaluating the malignancy of the newly diagnosed tumor, its contours, echogenicity, the nature of vascularization, ratio of antero-posterior dimensions and width of the tumor, as well as the presence of microcalcinates have a high diagnostic weight. The tumor size that determined by ultrasound and mammography does not have a high diagnostic weight.

Key words: breast canser, ultasonography, mammography, dopplerography

Language: Russian

Citation: Orujova IN, Asadov AS, Baxshiev BA (2018) DIAGNOSTIC VALUE OF SOME PARAMETERS OF RADİOLOGY METHODS IN BREAST CANCER. ISJ Theoretical \& Applied Science, 09 (65): $209-215$.

Soi: http://s-o-i.org/1.1/TAS-09-65-37 Doi: crostef https://dx.doi.org/10.15863/TAS.2018.09.65.37

\section{ДИАГНОСТИЧЕСКАЯ ЦЕННОСТЬ НЕКОТОРЫХ ПАРАМЕТРОВ ЛУЧЕВОЙ ДИАГНОСТИКИ ПРИ РАКЕ МОЛОЧНОЙ ЖЕЛЕЗЫ}

Аннотация: Цель нашей работы - определить диагностическую ценность радиологических методов, используемых для выявления рака молочной железы. В этой публикации мы представили результать исследования 76 женщин (18 - 79 лет), прошедших обследование по поводу рака молочной железы в онкологической клинике Азербайджанского Медицинского Университета в период с 2014 по 2017 год. Все пациенты прошли ультразвуковое исследование с допплерографией и рентгеновскую маммографию. Учитывая результаты наших исследований, можно сделать вывод о том, что при оценке злокачественности новообразований контуры, эхогенность, соотночение переднезадних размеров и ширины, характер васкуляризации опухоли, а также присутствие микрокальцинатов имеют высокий диагностический вес, при этом размер опухоли не обладает значимой диагностической иенностью.

Ключевые слова: рак молочной железы, маммография, УЗИ, допплерография
\end{abstract}

\section{Introduction}

Рак молочной железы (РМЖ) в структуре заболеваемости и смертности женского населения устойчиво занимает лидирующие позиции. Злокачественные новообразования молочной железы являются одной из актуальных медико-социальных проблем современной онкологии еще и потому, что поражают трудоспособное население, занимая одно из ведущих мест по утрате трудоспособности и причинам смерти от злокачественных новообразований у женщин [5, c. 28-32]. Немаловажную роль в исходе заболевания играет состояние овариальноменструальной функции женщины, ее возраст, состояние репродуктивной сферы, генетический фактор, особенности характера питания, наличие дисгормональных заболеваний молочных желез. 
Несмотря на то, что РМЖ чаще встречается в возрасте 55-65 лет, в последние годы наблюдается тенденция к возрастанию доли молодых женщин по отношению ко всему массиву больных РМЖ. Несомненно, что единственным способом борьбы с раком молочной железы, а также гарантом сохранения и улучшения качества жизни больного является своевременная диагностика [2, с. 2 ; 3, с. 52-54; 10 c. $515-520 ; 21$, с. 262-270]. Одним из основных показателей, определяющих прогноз онкологического заболевания, является степень распространенности опухолевого процесса на момент диагностики [4, с.359-362; 6, с. 9-10; 8, с. $29-30 ; 13$, с.773-839]. В этой связи на первый план выходят вопросы эффективного выявления РМЖ, и прежде всего, ранней диагностики на доклинической стадии [1, с. 664-667; 11,с. 23; 12 , с. $20-25 ; 16$, с.878-881; 17,с.1377-1385; 18]. Среди методов диагностики РМЖ, наиболее изученными являются рентгеновская маммография и ультразвуковое исследование (УЗИ) молочных желез [7, с.10-15; 9, с.53-57; 14 ,c. $75 ; 15$, c. $437-450 ; 19$, c. $26-34 ; 20$, c. $246-253$; 22,c. 16].

\section{Materials and Methods}

Целью нашей работы явилось определение диагностической ценности лучевых методов, используемых для выявления РМЖ. В настоящей работе были представлены результаты исследования 76 пациенток, проходивших обследование по поводу РМЖ в Онкологической Клинике Азербайджанского Медицинского Университета за период с 2014 по 2017 года. Возраст пациенток колебался от 18 до 79 лет. Всем больным были проведены ультразвуковое исследование с сочетанием допплерографии и рентгеновская маммография. Ультразвуковое исследование проводилось на аппарате MINDRAY D70 (Китай), а маммография при помощи аппарата SİEMENS MAMMOMAT İNSPIRATİON (Германия). При ультразвуковом обследовании оценивались переднезадние и поперечные размеры, контуры, эхогенность, эхоструктура, наличие и характер васкуляризации выявленного объемного новообразования, а также её локализация по квадрантам молочной железы. Принималось во внимание состояние регионарных подмышечных, надключичных, подключичных, парастернальных лимфатических узлов. Во время маммографии определялись контуры, размеры выявленной опухоли, также принимались во внимание её форма и наличие микрокальцинатов. После получения результатов плановых гистологических исследований нами проанализирована информативность и точность этих методов диагностики.

Из 76 обследованных пациенток у 48 женщин были обнаружены злокачественные, а у 28-доброкачественные новообразования. Выявленные новообразования имели правильные, лобулярные, неправильные и спикулярные контуры (Таб. 1). По данным УЗИ и рентгеновской маммографии у пациенток с РМЖ ровные контуры новообразования были обнаружены у 1, неровные - у 21, лобулярные -у 10 , а спикулярные - у 16 женщин. Только у одной пациентки, несмотря на правильные контуры, было морфологически подтверждено наличие злокачественного новообразования. Доброкачественные новообразования у 24 пациенток имели ровные, а у 4-лобулярные контуры.

\section{Характер новообразований, выявленных в молочной железе}

Таблица 1.

\begin{tabular}{|c|c|c|c|c|}
\hline \multicolumn{2}{|l|}{ Характер опухоли } & \multirow{2}{*}{$\begin{array}{l}\text { Доброкачественные } \\
\text { новообразования } \\
20\end{array}$} & \multirow{2}{*}{$\begin{array}{c}\text { 3локачественные } \\
\text { новообразования } \\
44\end{array}$} & \multirow{2}{*}{$\begin{array}{c}\text { Всего } \\
64 \\
\end{array}$} \\
\hline Размеры & $>10 \mathrm{MM}$ & & & \\
\hline & $\leq 10 \mathrm{MM}$ & 8 & 4 & 12 \\
\hline \multirow{4}{*}{ Контуры } & правильные & 24 & 1 & 25 \\
\hline & лобулярные & 4 & 10 & 14 \\
\hline & неправильные & - & 21 & 21 \\
\hline & спикулярные & - & 16 & 16 \\
\hline \multirow{3}{*}{ Эхогенность } & гиперэхогенная & 2 & - & 2 \\
\hline & гипоэхогенная & 9 & 44 & 53 \\
\hline & анэхогенная & 17 & 4 & 21 \\
\hline \multirow{2}{*}{$\begin{array}{l}\text { Отношение } \\
\text { АР и R }\end{array}$} & $\mathrm{AP}>\mathrm{R}$ & 4 & 36 & 40 \\
\hline & $\mathrm{AP}<\mathrm{R}$ & 24 & 12 & 36 \\
\hline \multirow{3}{*}{$\begin{array}{l}\text { Характер } \\
\text { васкуляризации при } \\
\text { допплерографии }\end{array}$} & Аваскулярный тип & 20 & 1 & 21 \\
\hline & Периферический тип & 7 & 11 & 18 \\
\hline & Центральный тип & 1 & 36 & 37 \\
\hline
\end{tabular}


АР-передне-задний размер опухоли

$\mathrm{R}$-ширина опухоли

Из данных, представленных в таблице 1, видно что для злокачественных опухолей молочной железы наиболее характерными признаками являются неправильные и спикулярные контуры, пониженная эхогенность, преобладание передне-заднего размера над шириной опухоли, диффузный (периферический и центральный) тип васкуляризации.

При УЗИ чувствительность формы контуров для оценки злокачественности опухоли составила
$97,9 \pm 2,1 \%$, а специфичность метода $-85,7 \pm 6,6 \%$ (Таблица 2.). Предсказательная полезность положительного результата была 92,2 $\pm 3,8 \%$, предсказательная полезность негативного результата - 96 $\pm 3,9 \%$. При этом отношение правдоподобия положительного результата составило 6,85 и оценивалось как хорошее, а негативного результата 0,02 и оценивалось как отличное. Общий диагностический вес теста был равен $93,4 \pm 2,8 \%$.

Диагностическая оценка некоторых параметров УЗИ при РМЖ ( в \%)

Таблица 2.

\begin{tabular}{|l|l|l|l|l|l|l|}
\hline Признак & $\mathrm{Se}$ & $\mathrm{Sp}$ & $\mathrm{pPV}$ & $\mathrm{nPV}$ & $\mathrm{LR}+$ & LR- \\
\hline Контуры & $97,9 \pm 2,1$ & $85,7 \pm 6,6$ & $92,2 \pm 3,8$ & $96,0 \pm 3,9$ & 6,85 & 0,02 \\
\hline Размеры & $91,7 \pm 4,0$ & $28,6 \pm 8,5$ & $68,8 \pm 5,8$ & $66,7 \pm 13,6$ & 1,28 & 0,29 \\
\hline $\begin{array}{l}\text { Отношение } \\
\text { АР и R }\end{array}$ & $75,0 \pm 6,3$ & $85,7 \pm 6,6$ & $90,0 \pm 4,7$ & $66,7 \pm 7,9$ & 5,25 & 0,29 \\
\hline Эхогенность & $91,7 \pm 4,0$ & $60,7 \pm 9,2$ & $80,0 \pm 5,4$ & $81,0 \pm 8,6$ & 2,33 & 0,14 \\
\hline Допплерография & $75,0 \pm 6,3$ & $96,4 \pm 3,5$ & $97,3 \pm 2,7$ & $69,2 \pm 7,4$ & 21,00 & 0,26 \\
\hline $\begin{array}{l}\text { Регионарные } \\
\text { лимфоузлы }\end{array}$ & $91,7 \pm 4,0$ & $57,1 \pm 9,4$ & $78,6 \pm 5,5$ & $80,0 \pm 8,9$ & 2,14 & 0,15 \\
\hline
\end{tabular}

$\mathrm{Se}$ - чувствительность - истинно (+) результаты

$\mathrm{Sp}$ - специфичность - истинно (-) результаты

$\mathrm{pPv}$ - предсказательная полезность $(+)$ результата nPV - предсказательная полезность (-) результата $\mathrm{LR}+-$ отношение правдоподобия (+) результата LR- - отношение правдоподобия (-) результата

Выявленные доброкачественные и злокачественные новообразования в зависимости от размеров мы разделили на 2 группы: 1) размеры $>10$ мм, 2) размеры $\leq 10$ мм. По данным УЗИ и рентгеновской маммографии у 44 пациенток размеры злокачественного образования составили больше $>10 \mathrm{Mм}$, а у 4-ёхразмеры были $\leq 10$ мм. По данным УЗИ 20 из доброкачественных новообразований имели размеры $>10$ мм, а у 8 размеры составляли $\leq 10$ мм, в то время, как при рентгеновской маммографии эти показатели были равны соответственно 23 и 5. Чувствительность размеров при оценке

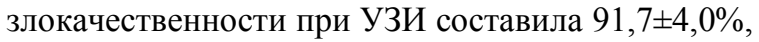
a специфичность метода $-28,6 \pm 8,5 \%$. Предсказательная полезность положительного результата составила 68,8 $\pm 5,8 \%$, предсказательная полезность негативного результата $-66,7 \pm 13,6 \%, \quad$ отношение правдоподобия положительного результата - 1,28 (оценивалось как непригодное), а негативного результата - 0,29 (оценивалось как посредственное). Общий диагностический вес теста составил $68,4 \pm 5,3 \%$.

При рентгеновской маммографии чувствительность размеров опухоли для оценки злокачественности составила $91,7 \pm 4,0 \%$, специфичность метода $-17,9 \pm 7,2 \%$, предсказательная полезность положительного результата - 65,7 $\pm 5,8 \%$, предсказательная

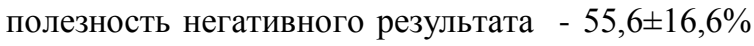
(Таблица 3). 


\begin{tabular}{l|lr|ll|ll} 
& ISRA (India) & $=\mathbf{1 . 3 4 4}$ & SIS (USA) & $=\mathbf{0 . 9 1 2}$ & ICV (Poland) & $=\mathbf{6 . 6 3 0}$ \\
Impact Factor: & ISI (Dubai, UAE) $=\mathbf{0 . 8 2 9}$ & PUHЦ (Russia) $=\mathbf{0 . 1 5 6}$ & PIF (India) & $=\mathbf{1 . 9 4 0}$ \\
& GIF (Australia) & $\mathbf{0 . 5 6 4}$ & ESJI (KZ) & $=4.102$ & IBI (India) & $=\mathbf{4 . 2 6 0}$ \\
& JIF & $=\mathbf{1 . 5 0 0}$ & SJIF (Morocco) & $=2.031$ & & \\
\hline
\end{tabular}

Таблица 3.

Диагностическая оценка некоторых параметров рентгеновской маммографии при РМЖ (в \%.)

\begin{tabular}{|l|l|l|l|l|l|l|}
\hline Признак & Se & Sp & Ppv & nPV & LR+ & LR- \\
\hline Контуры & $97,9 \pm 2,1$ & $85,7 \pm 6,6$ & $92,2 \pm 3,8$ & $96,0 \pm 3,9$ & 6,85 & 0,02 \\
\hline Размеры & $91,7 \pm 4,0$ & $17,9 \pm 7,2$ & $65,7 \pm 5,8$ & $55,6 \pm 16,6$ & 1,12 & 0,47 \\
\hline Микрокальцинаты & $43,8 \pm 7,2$ & $96,4 \pm 3,5$ & $95,5 \pm 4,4$ & $50,0 \pm 6,8$ & 12,25 & 0,58 \\
\hline
\end{tabular}

При этом отношение правдоподобия положительного результата составлило 1,12 и оценивалось как непригодное, а негативного результата - 0,47 и оценивалось как посредственное. Общий диагностический вес теста был равен $64,5 \pm 5,5 \%$.

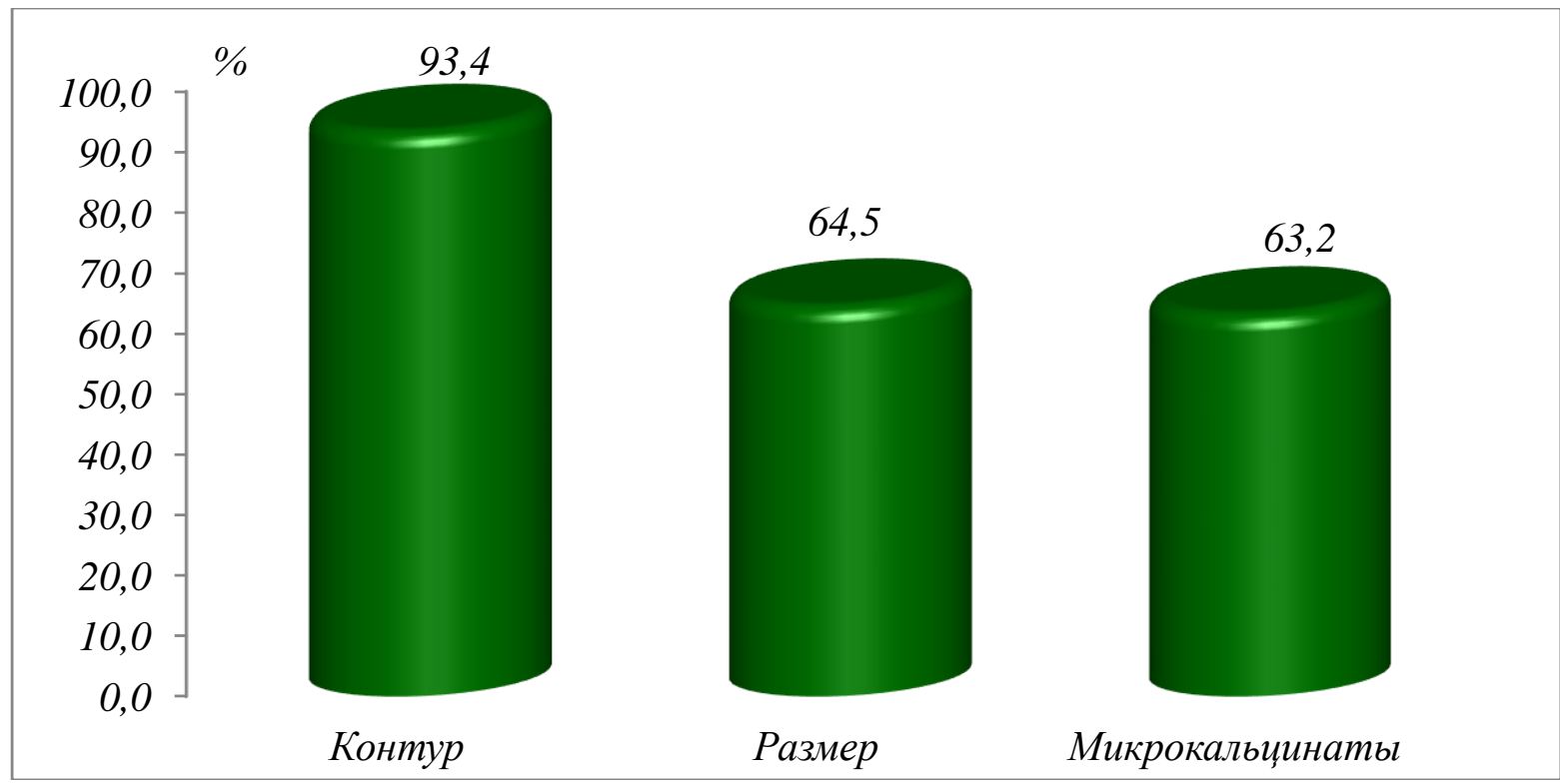

Рисунок 1. Общий диагностический вес параметров маммографии.

При УЗИ исследованиях, проведенных у 76 пациенток, выявленные опухоли имели различную эхогенность. Из выявленных злокачественных новообразований 4 были анехогенными, 44 гипоэхогенными, из доброкачественных 17- анэхогенными, 2 гиперэхогенными, 9 гипоэхогенными. Таким образом для злокачественных образований наиболее характерна гипоэхогенность. Чувствительность эхогенности при оценке злокачественности составила $91,7 \pm 4,0 \%, \quad$ a специфичность метода- $60,7 \pm 9,2 \%$. Предсказательная полезность положительного результата была $80,0 \pm 5,4 \%$, предсказательная полезность негативного результата - $81,0 \pm 8,6 \%$. Отношение правдоподобия положительного результата составило 2,3 и оценивалось как посредственное, а негативного результата - 0,14 и оценивалось как хорошее. Общий диагностический вес теста был равен $80,3 \pm 4,6 \%$. 


\begin{tabular}{|c|c|c|c|c|c|c|}
\hline Impact Factor: & $\begin{array}{l}\text { ISRA (India) } \\
\text { ISI (Dubai, UAE } \\
\text { GIF (Australia) } \\
\text { JIF }\end{array}$ & $\begin{array}{r}=1.344 \\
=0.829 \\
=0.564 \\
=1.500\end{array}$ & $\begin{array}{l}\text { SIS (USA) } \\
\text { PИHЦ (Russia) } \\
\text { ESJI (KZ) } \\
\text { SJIF (Morocco) }\end{array}$ & $\begin{array}{l}=0.912 \\
=0.156 \\
=4.102 \\
=2.031\end{array}$ & $\begin{array}{l}\text { ICV (Poland) } \\
\text { PIF (India) } \\
\text { IBI (India) }\end{array}$ & $\begin{array}{l}=6.630 \\
=1.940 \\
=4.260\end{array}$ \\
\hline
\end{tabular}

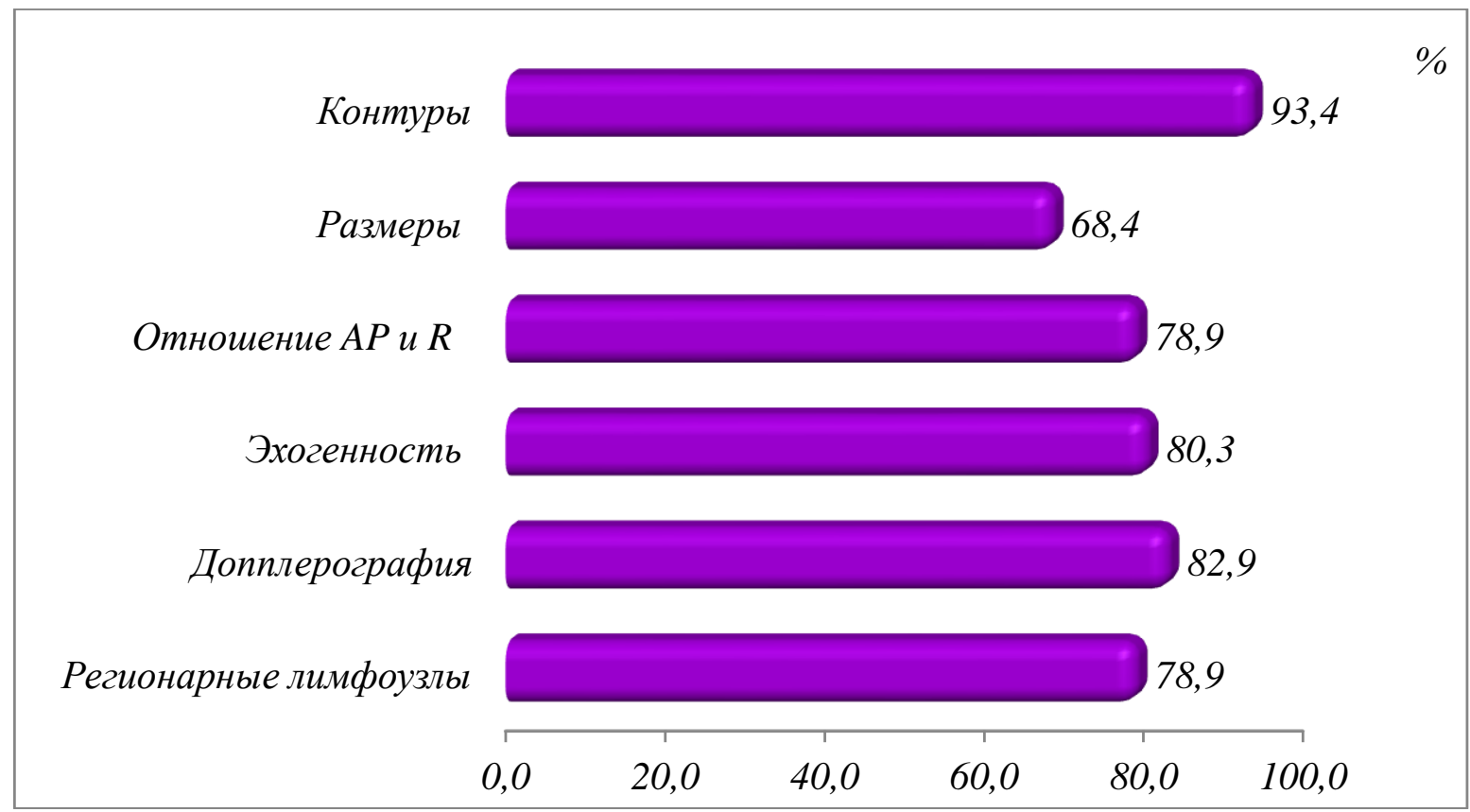

Рисунок 2. Общий диагностический вес параметров УЗИ.

У 36 пациенток со злокачественными новообразованиями, и у 4 с доброкачественными при УЗИ переднезадний размер опухоли превышал ширину, у 12 злокачественных и 24 доброкачественных новообразований переднезадний размер был меньше ширины. Чувствительность этого показателя при оценке злокачественности составила $75,0 \pm 6,3 \%$, а специфичность метода - $85,7 \pm 6,6 \%$. Предсказательная полезность положительного результата составила $90,0 \pm 4,7 \%$, предсказательная полезность негативного

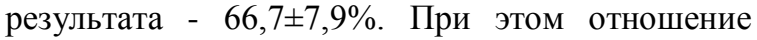
правдоподобия положительного результата составила 5,25 и оценивалось как хорошее, а негативного результата - 0,29 и оценивалось как посредственное. Общий диагностический вес

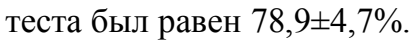

При допплерографии образований были получены различные результаты. Так у 36 злокачественных новообразований и у одного доброкачественного новообразования было выявлено интенсивное периферическое и центральное кровообращение. Периферический тип кровообращения был выявлен у 11 злокачественных, и у 7 доброкачественных новообразований. У одного злокачественного и у 20 доброкачественных новообразований кровообращения не обнаружено. Чувствительность допплерографии при оценке

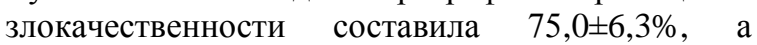
специфичность метода - 96,4 $\pm 3,5 \%$. Предсказательная полезность положительного результата была $97,3 \pm 2,7 \%$, предсказательная

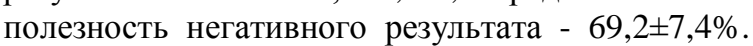
При этом отношение правдоподобия положительного результата составило 21,00 и оценивалось как отличное, а негативного результата - 0,26 оценивалось как посредственное. Общий диагностический вес теста был равен $82,9 \pm 4,3 \%$.

При рентгеновской маммографии у 21 из 48 пациенток с РМЖ и у 1 из 28 пациенток с доброкачественными изменениями были выявлены микрокальцинаты. Чувствительность микрокальцинатов при оценке злокачественности выявленной опухоли составила $43,8 \pm 7,2 \%$, а специфичность метода - $96,4 \pm 3,5 \%$. Предсказательная полезность положительного результата бала $95,5 \pm 4,4 \%$, предсказательная полезность негативного результата - 50,0土6,8\%. При этом отношение правдоподобия положительного результата составило 12,25 и оценивалось как отличное, а негативного результата - 0,58 и оценивалось как непригодное. Общий диагностический вес теста был равен $63,2 \pm 5,5 \%$.

При УЗИ измененные лимфоузлы были выявлены у 44 из 48 пациенток с РМЖ. Из них у 29 пациенток лимфоузлы были оценены как реактивные, у 8 как метастатические. У 7 пациенток были обнаружены и метастатические и реактивные лимфоузлы, что подтверждалось данными патоморфологического исследования клетчатки, удаленной во время операции. У 12 из 28 пациенток с доброкачественными новообразованиями были обнаружены реактивные лимфоузлы. Чувствительность лимфоузлов при оценке злокачественности составила $91,7 \pm 4,0 \%$, а специфичность метода $57,1 \pm 9,4 \%$. Предсказательная полезность положительного результата была 78,6 $\pm 5,5 \%$, 
предсказательная полезность негативного результата - $80,0 \pm 8,9 \%$, отношение правдоподобия положительного результата - 2,14 (оценивалось как посредственное), а негативного результата - 0,15 (оценивалось как хорошее). Общий диагностический вес теста составил $78,9 \pm 4,7 \%$.

\section{Conclusion}

Таким образом, принимая во внимание результаты проведенных нами исследований, можно прийти к выводу что, при оценке злокачественности выявленного новообразования высокую диагностическую значимость имеют его контуры (при УЗИ и маммографии), наличие микрокальцинатов (при маммографии), характер васкуляризации (при допплерометрии), а также эхогенность и соотношение передне-задних размеров и ширины опухоли (при УЗИ). Размеры опухоли определяемые при УЗИ и маммографии не обладают высоким диагностическим весом.

\section{References:}

1. Bukharin, D. G. (2011). Osobennosti mammograficheskoy vizualizatsii «malykh» form raka molochnoy zhelezy, rezvivshegosya na fone fibrozno-kistoznoy bolezni / D. G. Bukharin, E. M. Slonimskaya, S. A. Velichko, I.G. [i dr.] // Voprosy onkologii.. - T. 57, № 5. 2011 - S. 664-667.

2. Borisova, M. S. (2013). Rentgenovskaya mammografiya $\mathrm{v}$ diagnostike raka molochnoy zhelezy. [Elektronnyy resurs] / M. S. Borisova, N. V. Martynova, S. N. Bogdanov // Vestnik RNTsRR MZ. RF. -2013 - №13. Tom 3 Rezhim dostupa: http://vestnik.rncrr.ru/.

3. Burdina, L. M. (2003). Metody i sredstva sovremennoy rentgenodiagnostiki zabolevaniy molochnoy zhelezy: prakticheskoe rukovodstvo / L. M. Burdina, D. V. Makovkin. - Moskva: Strom , 2003 - 184 s.

4. Bus'ko, E. A. (2012). Vozmozhnosti sonoelastografii $\mathrm{v}$ diagnostike opukholey molochnykh zhelez razmerami do 2 sm. / E. A. Bus'ko, I. I. Semenov, V. F. Semiglazov // Voprosy onkologii.- T. 2, №3. 2012 -S. 359362.

5. Vysotskaya, I. V. (2010). Vozmozhnosti uluchsheniya skrininga raka molochnoy zhelezy / I. V. Vysotskaya //Opukholi zhenskoy reproduktivnoy sistemy- №4. 2010- S. 28-32.

6. Zabolotskaya N.V., Zabolotskiy V.S. (2010). Novye tekhnologii v ul'trazvukovoy mammografii. M.: OOO «Firma STROM». 2010-256 s.

7. Zapirova, S.B. (2009). Sovremennaya klassifikatsiya i osobennosti klinikorentgenosonopatomorfologicheskikh proyavleniy razlichnykh form mastopatii. / S.B. Zapirova, A.M. Bershchanskaya, N.L. Chazova, N.I. Rozhkova // Opukholi zhenskoy reproduktivnoy sistemy. Mammologiya / Onkoginekologiya. -№1-2. 2009- S. 10 - 15.

8. Zikiryakhodzhaev A.D., Letyagin V.P., Korzhenkova G.P., Volchenko A.A. (2008). Diagnosticheskaya tsennost' ul'trazvukovogo issledovaniya pri rake molochnoy zhelezy $\mathrm{u}$ zhenshchin molodogo vozrasta. // Rossiyskiy onkologicheskiy zhurnal. №2. -2008- S. 29-30.

9. Korzhenkova, G.P. (2008). Sovremennye podkhody k kompleksnoy luchevoy diagnostike raka molochnoy zhelezy. / G.P. Korzhenkova Obrazovatel'nyy kurs ESMO. M. - 2008. S.53-57.

10. Lindenbraten L.D., Korolyuk I.P.. (2000). Meditsinskaya radiologiya. // -2000-Moskva. «Meditsina». - S. 515-520.

11. Podol'skaya, E. A. (2011). Luchevye i tsitogistologicheskie sopostavleniya pri diagnostike nepal'piruemykh obrazovaniy molochnykh zhelez $\mathrm{u}$ zhenshchin rannego $\mathrm{i}$ zrelogo reproduktivnogo vozrasta: avtoref. dis. kand. med. nauk: 14.00.13 / Podol'skaya Ekaterina Aleksandrovna. - Obninsk, 2011$26 \mathrm{~s}$.

12. Serebryakova S.V., Trufanov G.E., Yukhno E.A. (2009). Magnitno-rezonansnaya semiotika raka molochnoy zhelezy. // Opukholi zhenskoy reproduktivnoy sistemy. Mammologiya / Onkoginekologiya. №3-4. 2009. S. 20-25.

13. Rumack C.M.,Wilson S.R., Charboneau J.W., Levine D. (2013). Tanısal Ultrasonografi. // GÜNEŞ TIP KITTABEVLERİ. 2013. S.773839.

14. American College of Radiology. (2003). BIRADS Breast imaging reporting abd data system. Breast imaging atlas: mammography, breast ultrasound, breast MR-imaging. Virginia. Reston. 2003. 268 p. 


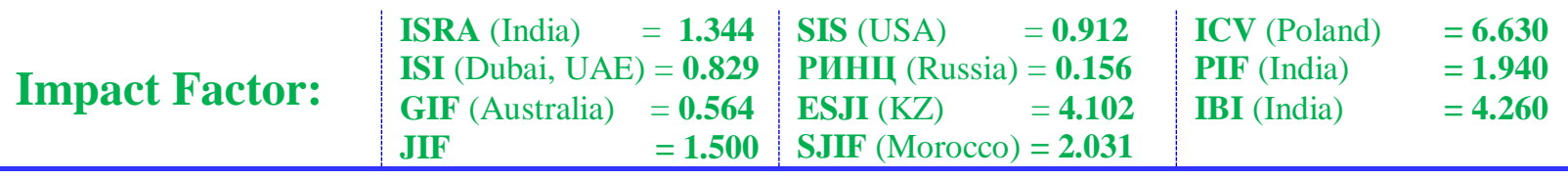

15. Cox R.F., Morgan M.P. (2013). Microcalcifications in breast cancer: Lessons from physiological mineralization. Bone. Vol. 53. №2. 2013. P. 437-450.

16. Aytac B., Sahsine T., Erturk F.Y. et al. (2013). Evaluation of incidence and histolopathological findings of breast lesions in reduction mammoplasty specimens: Uludag University experience. // J. Pak. Med. Assoc. Vol. 63. 2013. №7. R. 878-881.

17. Dana S. AL Mousa, MSc, Claudia MelloThoms, PhD, Elaine A. Ryan, PhD, Warwick B. Lee, PhD,Mariusz W. Pietrzyk, PhD, Warren M. Reed, PhD, Robert Heard, PhD, Ann Poulos, PhD,Jennifer Tan, MD, Yanpeng Li, MSc, Patrick C. Brennan, PhD. (2014).Mammographic Density and Cancer Detection: Does Digital Imaging Challenge our Current Understanding// Acad Radiol; 2014. 21:1377-1385.

18. Gøtzsche PC, Nielsen M. (2009).Screening for breast cancer with mammography. Cochrane
Database Syst Rev. Oct 7;(4):CD001877. 2009. doi: 10.1002/14651858.CD001877.pub3.

19. Hafslund B, Espehaug B, Nortvedt MW. (2012). Effects of false-positive results in a breast screening program on anxiety, depression and health-related quality of life. Cancer Nurs. 2012.p26-34.

20. Hendrick R.E. (2010). Radiation Doses and Cancer Risks from Breast Imaging Studies //Radiology. - №257. - 2010.P.246-253.

21. Hamed S.T., Abdo M.H., Ahmed H.H. (2008). Breast discharge: ultrasound and Doppler evaluation. // J. Egypt Natl. Canc. Inst. Vol. 20. 2008. №3. P. 262-270.

22. Kerlikowske K, Zhu W, Hubbard RA, Geller B, Dittus K, Braithwaite D, et al; (2013). Breast Cancer Surveillance Consortium. Outcomes of screening mammography by frequency, breast density, and postmenopausal hormone therapy. JAMA Intern Med.; 2013. 173:807-16. 